\title{
Ultrasonic Non-Invasive Method for Relative Changes Measurements of Intima-Media Thickness in Artery Walls
}

\author{
Z. Trawiński*, J. WóJCik, T. POWAŁOWSKI AND
}

\author{
P. GutKiewicz
}

Institute of Fundamental Technological Research, Polish Academy of Sciences Świętokrzyska 21, 00-049 Warsaw, Poland

\begin{abstract}
The aim of this paper is to present the new method for relative changes measurements of intima-media thickness in the common carotid artery wall. The numerical solver was created for calculation of the fields of ultrasonic beams and scattered fields under different boundary conditions and different angles of penetration of ultrasonic beams with respect to the position of the arterial wall. The cylindrical model of the artery was changing the radius and thickness of the wall under cyclic variation of blood pressure. The presented method was verified on a pipe made of latex. The paper describes also the initial results of examinations of the intima-media thickness. The good agreement for the angle dependence and the perpendicular ultrasonic beam displacement from the longitudinal axis of the artery segment between the numerical calculation and experimental results was obtained for different artery diameters.
\end{abstract}

PACS numbers: 43.25. Ed, 43.25.Zx, 43.35.Yb, 43.35.Bf, 43.38.Fx, 43.58.Ta, 43.60.Tj

\section{Introduction}

The pathological process of atherosclerosis development and its connection with alterations that occur in walls of blood vessels present a matter of interest for numerous scientific and clinical centers worldwide. In case of non-invasive investigations, the ultrasonic measurements for momentary diameters of arteries over the entire cardiac cycle serve as the basis enabling to determine elasticity of arterial walls. Maximum and minimum values for the vessel diameter are associated with respective systolic and diastolic blood pressures measured by a sphygmomanometer. Based on the above measurements, the elasticity factors of the arterial wall are

*corresponding author; e-mail: ztraw@ippt.gov.pl 
determined $[1,2]$. In case of non-invasive ultrasonic measurement, reproducibility of the obtained results is an extremely important parameter, since it is used to define sensitivity of the diagnostic tool [3-6]. The major objective of the thesis was to develop a mathematical model that would be capable of describing spatial and time-dependent distribution of an ultrasonic beam that is emitted by a piezoelectric ring transducer and then backscattered on cylindrical surfaces of the walls in artery models [7-10]. The developed model was tested for results of experiments when an elastic pipe was immersed in water. The investigations were carried out using the VED equipment, designed and constructed in the Ultrasonic Department of the Institute of Fundamental Technological Research of the Polish Academy of Sciences, purposefully dedicated for elasticity examination of arterial walls in human body.

\section{Physical model}

With use of non-dimensional variables, the equation that defines the propagation of sonic waves in a homogeneous (with undisturbed parameters of the material) non-linear and absorbing medium, can be expressed by the following equation [4]:

$$
\Delta P-\partial_{t t} P-2 \partial_{t} \boldsymbol{A} P+q \beta \delta_{t t}(P)^{2}=0,
$$

where $\boldsymbol{A} P \equiv A(t) \otimes P(\boldsymbol{x}, t), A(t)=F^{-1}[a(n)], P(\boldsymbol{x}, t)$ is the pressure in the $3 \mathrm{D}$ coordinate system $\boldsymbol{x}$ at the moment of time $t ; \boldsymbol{A}$ is a convolution-type operator that defines absorption; $q$ is the Mach number (in our case the Mach number is calculated for velocities on the surface of the disturbance); $\beta \equiv(\gamma+1) / 2$; $\gamma \equiv B / A+1$ or $\gamma$ - adiabatic exponent, $n \equiv f / f_{0}$ - non-dimensional frequency; $f, f_{0}$ - respectively: frequency and characteristic frequency; $a(n)$ - the small signal coefficient of absorption, $\boldsymbol{A}=F^{-1}[a(n)], F[$.$] — the Fourier transform.$

For the medium with disturbed materials parameters the equation of the scattered field $P^{\text {sc }}$ can be developed from the formula (1) (more details see [5]) and takes the form

$$
\Delta P^{\mathrm{sc}}-\partial_{t t} P^{\mathrm{sc}}-2 \partial_{t} \boldsymbol{A} P^{\mathrm{sc}}=-\Pi \partial_{t t}\left(P^{\mathrm{sc}}+P^{\mathrm{in}}\right),
$$

where $P^{\text {in }}$ - incident field which fulfils Eq. (1), $\Pi(\boldsymbol{x}) \equiv 1-1 / c_{r}^{2}-$ scattering potential, $c_{r}^{2}$ - disturbed dimensionless sound velocity.

\section{Solver}

Construction of a solver for backscattered fields is the fundamental issue for setting up a numerical model of an experiment that is aimed to reflect real situations that occur in ultrasonography practice [11-14]. The solver that we constructed is composed of three parts:

1) Solver for the incident field. It is the solver that bases on codes JWNUT2D and JWNUT3D [6], which we have been using for many years. The first code solves the equation in the axially symmetrical cases, the second one is applicable to whichever one-sided boundary conditions. 
2) Solver for the backscattered field. It is the tool that is able to calculate parameters of backscattered fields and their pressures on the detector surface, whereas the tool uses numerically determined incident field and information on geometrical and material parameters of the target as the basis for calculations.

3) Simulator of the electronic receiver channel that is used for calculation of pulse responds $h(t)$ of this unit. Distribution of pressure on the surface of the probe is averaged over the entire probe surface (the theory of piezoelectric phenomena says that electric signals at probe output are proportional to the aforementioned average value).

$$
P_{\mathrm{E}}(t)=\frac{C_{p}}{S} \int_{S(\boldsymbol{x})} P^{\mathrm{sc}}(S(\boldsymbol{x}), t) A p(S(\boldsymbol{x})) \mathrm{d} S,
$$

where $C_{p}$ is parameter characteristic of probe (i.e. connected with piezoelectric constants), $S(\boldsymbol{x})$ denotes a point on the transducer surface, $S$ stands for the transducer surface area and $A p(S(\boldsymbol{x}))$ is the apodization function for the transducer surface. In this study $P_{\mathrm{E}}(t)$ is referred to as the echo. The RF signal $P_{\mathrm{RF}}(t)$ represents a single line of scanning and is calculated as follows:

$$
P_{\mathrm{RF}}(t)=h(t) \otimes P_{\mathrm{E}}(t), \quad h=F^{-1}[H(n)],
$$

where $H(n)$ is the system transmittance.

\section{Results}

Experimental setup is shown in Fig. 1. The research was carried out for a pipe made of latex, with internal diameter of $5 \mathrm{~mm}$ and wall thickness of $1.25 \mathrm{~mm}$. The investigations employed the VED ultrasonic apparatus, designed by the authors for the purpose of examining the elasticity of arteries. The frequency of the

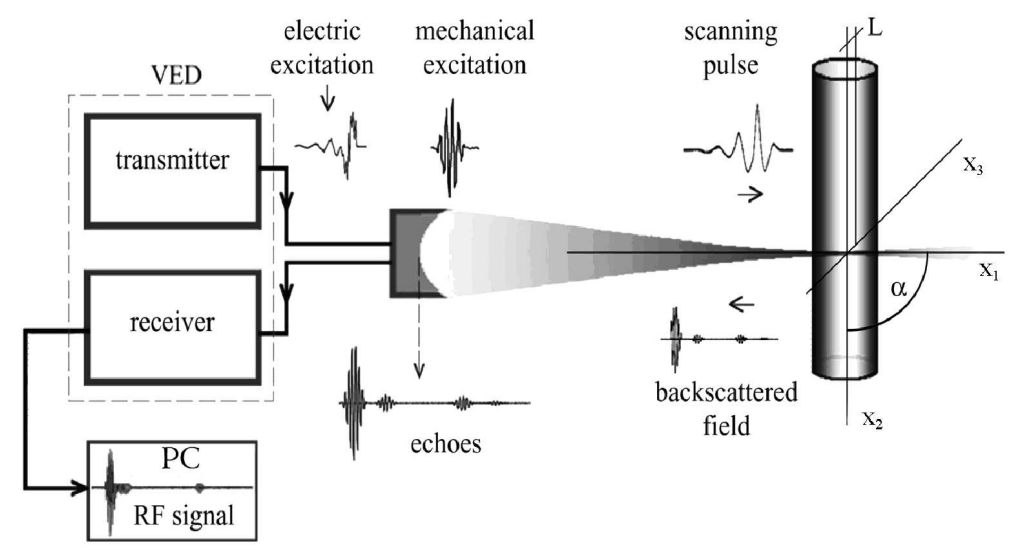

Fig. 1. Experimental setup. 

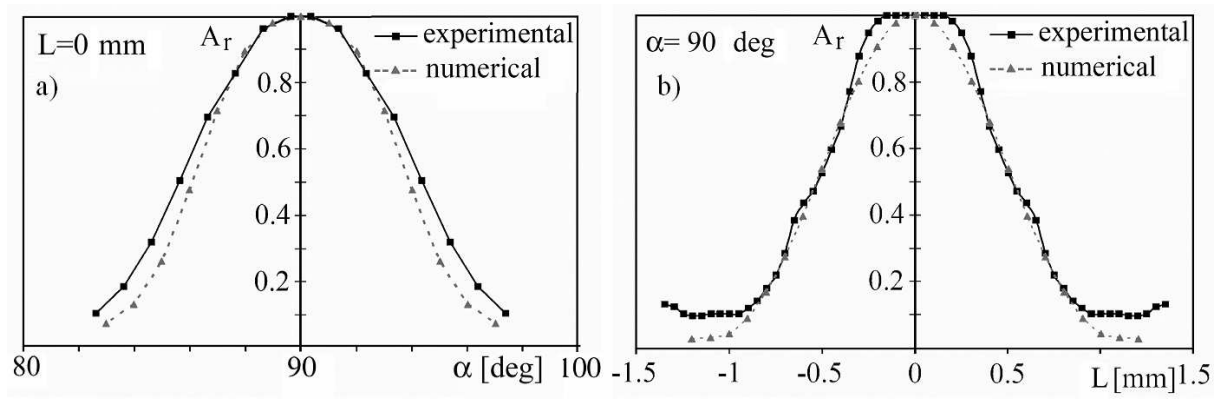

Fig. 2. (a) Changes of the maximal RF-signal amplitude as a function of angle $\alpha$ and (b) the transversal displacement $L$ of the ultrasonic beam axis $x_{1}$, with respect to the arterial model axis $x_{2}$ (see Fig. 1) for phantom diameter $5 \mathrm{~mm} . A_{r}$ - maximal RF-signal amplitude with respect to the maximal RF-signal amplitude for the angle $\alpha=90^{\circ}$ and transversal displacement $L=0 \mathrm{~mm}$.
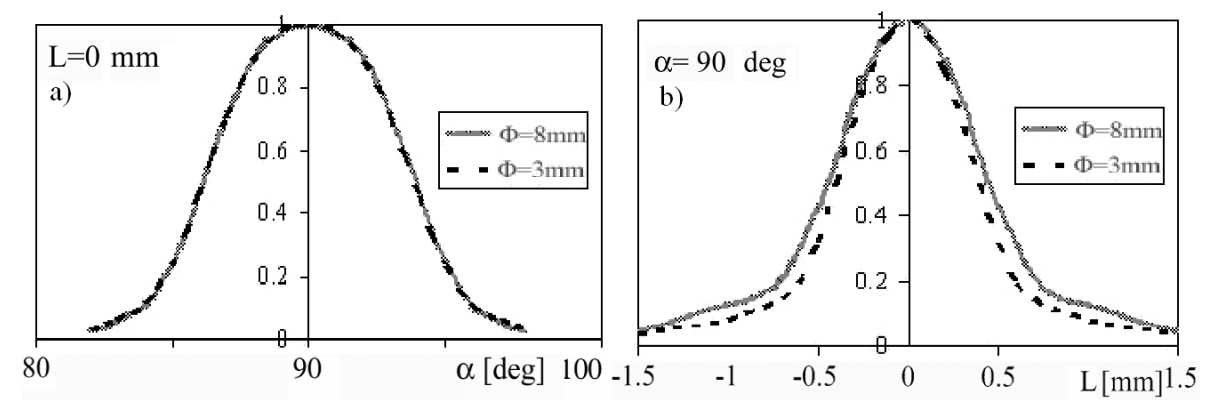

Fig. 3. Results of calculations for phantom diameter $8 \mathrm{~mm}$ and $3 \mathrm{~mm}$ : (a) for the transversal displacement $L=0 \mathrm{~mm}$; (b) for the angle $\alpha=90^{\circ} . A_{r}-$ maximal $\mathrm{RF}$-signal amplitude with respect to the maximal RF-signal amplitude.

transmitted ultrasound was $6.75 \mathrm{MHz}$. During the performed research the front surface of the pipe wall was positioned in the focus of the ultrasonic probe. The $\mathrm{RF}$ electric signal, $P_{\mathrm{RF}}(t)$, corresponding to echoes reflected by the pipe walls, was recorded at the output of the RF receiver of VED by means of the PC $100 \mathrm{MHz}, 14$-bit Signatec data acquisition card, type PDA-14. To highlight relationships between target (pipe) dimensions and wavelength of the echoed signals (both acoustic waves and corresponding electric wave forms) the scales were converted all the time into 3D ones and expressed in millimeters. Signal amplitudes were presented as relative values. Results for calculations and measurements are presented in Figs. 2 and 3. 


\section{Conclusions}

Comparison between the results that were obtained from numerical calculations and from measurements (Figs. 2 and 3) serves as a proof that the numerical model that was developed by our own enables simulation of the experiments with a good coherence, which was the actual objective of the study. Moreover, the developed solver makes is possible to acquire information about the ultrasonic signal on every stage of its processing, with consideration to transfer functions of all the components down the receiving path, i.e. the ultrasonic probe, $\mathrm{RF}$ amplifier, etc. It is the matter of high importance when the designing process of measurement equipment is to be optimized.

\section{Acknowledgments}

The current investigation is a part of a research project of the Ministry of Higher Education and Science No. 3 T11E 01129.

\section{References}

[1] L.H. Peterson, R.E. Jensen, J. Parnell, Circ. Res. 8, 622 (1960).

[2] W.A. Riley, R.W. Barnes, H.M. Schey, Prev. Med. 13, 169 (1984).

[3] R. Reneman, T. Van Merode, P. Hick, A.M.M. Mutytjens, A.P.G. Hoeks, Ultrasound Med. Biol. 12, 465 (1986).

[4] T. Van Merode, P.J. Brands, A.P.G. Hoeks, R.S. Reneman, J. Hypert. 11, 171 (1993).

[5] T. Powałowski, B. Peńsko, Arch. Acoust. 13, 109 (1988).

[6] T. Kawasaki, S. Sasayama, S. Yagi, T. Asakawa, T. Hirai, Cardiov. Res. 21, 678 (1987).

[7] T. Szymoński, T. Powałowski, Z. Trawiński, M. Łapiński, Acta Angiol. 3, 83 (1997).

[8] K. Wojtowicz, Mol. Quant. Acoust. 28, 293 (2007).

[9] J. Wójcik, J. Acoust. Soc. Am. 104, 2654 (1998).

[10] J. Wójcik, Arch. Acoust. 29, 607 (2004).

[11] Z. Trawiński, T. Powałowski, Arch. Acoust. 31, 4 S., 29 (2006).

[12] P. Gibinski, E. Maciak, Z. Opilski, T. Pustelny, A. Sieron, M. Urbanczyk, T. Woznica, Mol. Quant. Acoust. 28, 217 (2007).

[13] T. Powałowski, Z. Trawiński, J. Wójcik, Mol. Quant. Acoust. 28, 279 (2007).

[14] Z. Trawiński, T. Powałowski, P. Gutkiewicz, Arch. Acoust. 32, 4 S., 135 (2007). 